\title{
Genetic diversity in migratory bats: Results from RADseq data for three tree bat species at an Ohio windfarm
}

Michael G Sovic, Bryan C Carstens, H. Lisle Gibbs

Genetic analyses can identify the scale at which wildlife species are impacted by human activities, and provide demographic information useful for management. Here, we use thousands of nuclear DNA genetic loci to assess whether genetic structure occurs within Lasiurus cinereus (Hoary Bat), L. borealis (Red Bat), and Lasionycteris noctivagans (SilverHaired Bat) bats found at a wind turbine site in Ohio, and to also estimate demographic parameters in each of these three groups. Our specific goals are to: 1) demonstrate the feasibility of isolating RADseq loci from these tree bat species, 2) test for genetic structure within each species, including any structure that may be associated with time (migration period), and 3) use coalescent-based modeling approaches to estimate geneticallyeffective population sizes and patterns of population size changes over evolutionary timescales. Thousands of loci were successfully genotyped for each species, demonstrating the value of RADseq for generating polymorphic loci for population genetic analyses in these bats. There was no evidence for genetic differentiation between groups of samples collected at different times throughout spring and fall migration, suggesting that individuals from each species found at the wind facility are from single panmictic populations. Estimates of present-day effective population sizes varied across species, but were consistently large, on the order of $10^{5}-10^{6}$. All populations show evidence of expansions that date to the Pleistocene. These results, along with recent work also suggesting limited genetic structure in bats across North America, argue that additional biomarker systems such as stable-isotopes or trace elements should be investigated as alternative and/or complementary approaches to genetics for sourcing individuals collected at single wind farm sites. 
1 Genetic diversity in migratory bats: Results from RADseq data for three tree bat species at an

2 Ohio windfarm.

3

4 Michael G. Sovic ${ }^{1,2}$, Bryan C. Carstens ${ }^{1}$, and H. Lisle Gibbs ${ }^{1,2}$

$5{ }^{1}$ Department of Evolution, Ecology and Organismal Biology, The Ohio State University,

6 Columbus $\mathrm{OH} 43210$, USA

7 2Ohio Biodiversity Conservation Partnership, The Ohio State University, Columbus OH 43210,

8 USA

9

10 Corresponding Author:

11 Michael G. Sovic

12300 Aronoff Laboratory, 318 W. 12 ${ }^{\text {th }}$ Avenue, Columbus OH 43210, USA

13 sovic.1@,osu.edu

14

15

16

17

18

19

20

21

22 


\section{Abstract}

Genetic analyses can identify the scale at which wildlife species are impacted by human activities, and provide demographic information useful for management. Here, we use thousands

27 of nuclear DNA genetic loci to assess whether genetic structure occurs within Lasiurus cinereus

28 (Hoary Bat), L. borealis (Red Bat), and Lasionycteris noctivagans (Silver-Haired Bat) bats found

29 at a wind turbine site in Ohio, and to also estimate demographic parameters in each of these three

30 groups. Our specific goals are to: 1) demonstrate the feasibility of isolating RADseq loci from

31 these tree bat species, 2) test for genetic structure within each species, including any structure

32 that may be associated with time (migration period), and 3) use coalescent-based modeling

33 approaches to estimate genetically-effective population sizes and patterns of population size

34 changes over evolutionary timescales. Thousands of loci were successfully genotyped for each

35 species, demonstrating the value of RADseq for generating polymorphic loci for population

36 genetic analyses in these bats. There was no evidence for genetic differentiation between groups

37 of samples collected at different times throughout spring and fall migration, suggesting that

38 individuals from each species found at the wind facility are from single panmictic populations.

39 Estimates of present-day effective population sizes varied across species, but were consistently

40 large, on the order of $10^{5}-10^{6}$. All populations show evidence of expansions that date to the

41 Pleistocene. These results, along with recent work also suggesting limited genetic structure in

42 bats across North America, argue that additional biomarker systems such as stable-isotopes or

43 trace elements should be investigated as alternative and/or complementary approaches to

44 genetics for sourcing individuals collected at single wind farm sites. 


\section{Introduction}

Species that migrate over large spatial scales can spend substantial amounts of time in

transit between breeding and wintering locations, and experience substantial impacts from pointsource anthropogenic factors during this period (Rappole \& McDonald 1994; Faaborg et al. 2010). For example, migrating bats and birds experience mortality as a result of collisions with wind turbines and cell phone towers, habitat loss, exposure to disease, and global climate change (Jonzén, Hedenström, \& Lundberg 2007; Altizer, Bartel \& Han 2011; Loss, Will \& Marra 2013). Identifying the scale and magnitude of these geographically limited factors can be important in assessing the scale of these impacts on species of conservation concern.

Specifically, wind turbines have been shown to cause a substantial number of mortalities to bat species (Johnson 2005; Kunz et al. 2007), and particularly impact migratory tree bats (Arnett et al. 2007; Cryan \& Brown 2007). Thermal imaging studies demonstrate that bats may be attracted to turbines (Horn, Arnett \& Kunz 2008), and the low-pressure pockets of air produced by these turbines may lead to barotrama (Kunz et al. 2007; Baerwald et al. 2008). Regardless of the cause of death, a study produced by the National Renewable Energy Lab suggests that turbines will cause tens of thousands of fatalities among migratory tree bat species by the year 2020 (Kunz et al. 2007). Given this magnitude of potential impact, researchers and agencies are actively seeking to understand and mitigate, to the extent possible, the impact of wind farms on bat species.

Several hypotheses have been offered to explain the attraction of bats to wind energy installations (reviewed by Kunz et al. 2007). It may be that their insect prey are more abundant in the clearings created by these facilities, or that sound produced by the turbines could either attract or disorient the bats. Using bat detectors as monitors, recent research demonstrated that 
70 the activity of tree bats increases dramatically at tall structures during migrations for reasons

71 other than foraging (Jameson \& Willis 2014). This supports earlier suggestions that bats mistake

72 the installations for tall trees, which are likely used as lekking points by some species

73 (particularly Lasiurus; Cryan 2008). It may be that these facilities function as aggregation points

74 for migratory individuals, as previous work has suggested that migratory bats are impacted more

75 than resident populations (Johnson et al. 2003).

In some migratory birds, genetically distinct populations appear to use geographically

77 restricted flyways (Boulet, Gibbs \& Hobson 2006; Ruegg et al. 2014). Thus, the impact of

78 mortality at a given stopover site will affect a geographically restricted population, or set of

populations. Whether this pattern also occurs in other migratory animals such as bats is unknown.

A first step in establishing whether genetically-based biomarkers can detect similar patterns in bats is to conduct genetic assays of migratory individuals at specific sites where bats suffer high mortality. Identifying patterns of genetic structure would be consistent with multiple geographically and genetically distinct populations migrating through a single site. An association between this structure and time may further reflect temporal differences in migration patterns among any genetically distinct groups.

In addition to evaluating patterns of structure, genetic data can also be used to assess

87 demographic trends and estimate population parameters that may be important in a conservation context. Genomic-scale SNP data, such as that generated with restriction site associated DNA sequencing (RADseq, Davey \& Blaxter 2011), along with novel methods that efficiently analyze large SNP datasets (i.e. Gutenkunst et al. 2009, Excoffier et al. 2013), provide powerful options

91 for testing among alternative demographic scenarios and estimating associated population

92 parameters. Doing so can identify patterns of population size change over time that may reflect 
93 anthropogenic impacts, or quantify parameters such as effective population size $(\mathrm{Ne})$ that may

94 indicate a population's potential to adapt to future environmental changes, or to suffer from

95 factors such as inbreeding depression that are related to levels genetic diversity.

96 Lasiurus cinereus (Hoary Bat), L. borealis (Red Bat), and Lasionycteris noctivagans

97 (Silver-haired Bat) are three species of tree bats that are distributed throughout North America,

98 and are active in the Midwest between April and November (Kurta 1995). The migratory habits

99 of these three species make them especially susceptible to effects of wind facilities, and indeed

100 high levels of mortality have been documented for each of these species at wind farms (Kunz et

101 al. 2007). Previous work using microsatellite and mitochondrial DNA data assessed genetic

102 variation in two of these species (L. cinereus and L. borealis), and found no evidence for

103 population-level differentiation in either group (Vonhof \& Russell 2015; Korstian, Hale \&

104 Williams 2015). Large SNP datasets such as those described above offer an alternate source of

105 genetic markers that may provide additional power to detect more subtle patterns of

106 differentiation, and to estimate demographic patterns and/or parameters with greater precision.

107 Here, we use RADseq data to determine levels and patterns of genetic diversity in samples of

108 these three bat species collected from a wind facility in Ohio, to assess the extent to which the

109 genetic diversity in the samples is structured by the time of death, to test for evidence of trends in

110 population size over time, and to estimate demographic parameters using coalescent-based

111 models.

112

113 Methods

114 Sample collection 
116 by the agency at an Ohio wind facility during the spring, summer and fall of 2012. Samples used

117 for the genetic analyses presented here included 44 Hoary bats (Lasiurus cinereus), 37 Silver-

118 haired bats (Lasionycteris noctivagans), and 53 Eastern Red bats (Lasiurus borealis). While

119 samples from both Lasiurus species were collected throughout the season, Lasionycteris

120 noctivagans were sampled either early or late in the season. A small wing biopsy was obtained

121 from each sample and placed in $95 \% \mathrm{EtOH}$ until further processing. DNA extraction was

122 conducted using a Qiagen DNA Blood and Tissue kit (Qiagen) following standard protocols.

\section{Library Preparation}

125 Restriction enzymes EcoRI and SbfI were used to digest genomic DNA, and double-

126 digest RADseq libraries were generated for each sample (Peterson et al. 2012). Libraries were

127 pooled in equimolar amounts prior to sequencing, and run in single read 75-bp runs on an

128 Illumina HiSeq 2500. Details of the library preparation methods are provided as supplemental

129 material.

Bioinformatics processing

Denovo locus identification and genotyping was performed separately for each species with AftrRAD version 4.1 (Sovic, Fries \& Gibbs 2015), which can assemble RADseq loci in a

134 computationally efficient manner, while accounting for indel variation among alleles. AftrRAD

135 analyses were carried out with default parameters, with the exception that we trimmed SNPs

136 occurring beyond read position 55 (after also trimming the in-line barcodes and restriction

137 recognition sequence at the beginning of reads) to remove artifactual SNPs that accumulate 
138 toward the ends of reads (see Sovic, Fries \& Gibbs 2015). The default parameters used include a

139 relatively conservative threshold for initial quality filtering that removes all reads containing

140 quality scores $<20$. To reduce possible effects of allele drop out (Arnold et al. 2013; Gautier et al.

141 2013), we conservatively retained for analyses only those loci scored in all of the samples in

142 each dataset.

143

144 Data analysis

145

146 Genetic Isolation by Mortality Time

147 We conducted two analyses on all three species to assess the extent to which genetic

148 diversity was clustered as a function of the time of collection. First, we conducted an analysis of

149 molecular variance (Weir \& Cockerham 1984; Excoffier, Smouse \& Quattro 1992; Weir 1996)

150 using Arlequin 3.5 (Excoffier \& Lischer 2010) in order to assess whether genetic variance was

151 partitioned across time. Samples were divided into two (L. noctivagans, L. cinereus) or three (L.

152 borealis) groups containing samples that were collected at similar times throughout the year (Fig.

153 1), and the genetic variance was decomposed into either among-group or within-group variance.

154 We then compared these values to the total variance and assessed the statistical significance of

155 our findings using randomized permutations of the data. AMOVAs are commonly conducted in

156 order to assess the importance of geography for structuring genetic variation. Our analysis here is

157 conducted in a similar manner, but using time rather than space as the physical axis of interest.

158 Second, we tested the relationship between genetic diversity and the time of sample

159 collection in the absence of any clustering. This analysis, which we refer to as an isolation-by-

160 time analysis, is essentially an isolation by distance analysis, but with a temporal matrix that 
161 consisted of a count of the number of days between the time of collection for any given pair of

162 samples. A Mantel test, implemented in GenePop (Raymond \& Rousset 1995; Rousset 2008)

163 was used to conduct the isolation by time analysis.

164 In addition, we analyzed the data with STRUCTURE (Pritchard, Stephens \& Donnelly

165 2000), which performs clustering based on the genotype data alone, and can therefore identify

166 genetic structure that may be partitioned by any factor, including time, geography, etc. For each

167 of the three species we evaluated possible $\mathrm{K}$ values ranging from 1-5 (5 runs for each $\mathrm{K}$ value)

168 under a model of admixture and correlated allele frequencies. The Markov chain was run for 5.0

$169 \times 10^{5}$ steps per analysis, with a burn-in of $1.0 \times 10^{5}$. Log likelihood values were averaged across

170 runs for each $\mathrm{K}$ value, and these values were evaluated along with patterns of individual

171 assignment to clusters to draw inference regarding the optimal $\mathrm{K}$ value for each dataset.

172

\section{Estimation of population and demographic parameters}

174 Finally, given that the analyses outlined above did not provide any strong evidence for

175 genetic structure within each species (see below), we generated single population folded site

176 frequency spectra for each of the datasets using scripts associated with AftrRAD, and used these

177 to assess historical demographic patterns and estimate associated parameters in the three species.

178 Each single-population site frequency spectrum was generated using a maximum of one SNP per

179 RADseq locus by using the "unlinked" flag in AftrRAD to select the first biallelic SNP at loci

180 that contained multiple SNPs. This was done in an effort to alleviate any effects of tight linkage

181 among SNPs at such loci. The number of monomorphic sites in the site frequency spectrum was

182 scaled based on the proportion of linked SNPs that were removed from the dataset

183 ("MonoScaled" flag in AftrRAD). As a sensitivity analysis, additional site frequency spectra 
184 were generated that included all sites in the dataset. For all three species, the same demographic

185 model was chosen as optimal regardless of whether linked SNPs were included or not. Parameter

186 estimates were also comparable between the datasets, suggesting that the conclusions related to

187 the demographic modeling were not highly sensitive to patterns of linkage.

188 We used FastSimCoal252 (Excoffier et al. 2013) to calculate the likelihood of the

189 observed site frequency spectra under alternative demographic models, and we compared these

190 models with AIC. The first model represented a constant population size, and included effective

191 population size as the only estimated parameter (Fig. S1A). The second model allowed for

192 population growth or decline at a constant rate, followed (backwards in time) by constant

193 population size. This model estimated the current population size, the population size prior to the

194 growth or decline, and the time at which the growth/decline event began (Fig. S1B). All

195 parameters were selected from a uniform distribution of $10-1,000,000$ that was not bounded on

196 the upper end of the distribution. For each model, we performed 100 independent runs of fsc 252

197 (30 ECM cycles, 50,000 simulations per run), and we chose the run with the highest likelihood

198 for each model for AIC model selection. Following Excoffier et al. (2013), we used point

199 estimates for parameters from the optimal model/run for each species to perform parametric

200 bootstrapping, and we used these bootstrapped datasets to generate confidence intervals for the

201 estimated parameters. Parameters were estimated assuming an average rate of $2.5 \times 10^{-8}$

202 mutations per nucleotide site per generation, as reported by Nachman \& Crowell (2000) for

203 humans. To determine the sensitivity of our analyses to the value of the mutation rate parameter,

204 we also performed model choice and parameter estimation analyses using a rate of $2.366 \times 10^{-9}$

205 mutations per nucleotide site per generation, which was estimated by Ray et al. (2008) from

206 limited intron data for vespertilionid and miniopterid bats. The specific value of the mutation rate 
207 did not change inferences regarding model selection, but did influence parameter estimates at a

208 magnitude comparable to the difference in the two assumed mutation rates. Specifically,

209 effective population size estimates were approximately 10x larger, and dates of historical events

210 were approximately $10 \mathrm{x}$ older with the lower mutation rate. We report parameter estimates based

211 on the value of $2.5 \times 10^{-8}$ mutations per nucleotide site per generation for two reasons. First, this

212 value leads to more conservative estimates in parameters such as $\mathrm{Ne}$ from a conservation

213 perspective. Second, this value is comparable to other estimates of genome-wide mutation rates

214 that have been calculated directly from whole-genome data (Keightley 2012), suggesting that it

215 may be a better estimate of the true mutation rate for a mammal. Effective population size

216 estimates are reported throughout the text as the number of chromosomes in the population. As

217 such, the number of diploid individuals represented by these numbers would be equal to half

218 these values. Finally, overall levels of heterozygosity were calculated for each dataset using

219 AftrRAD.

220

221 Results

222 RADseq Data

223 Nearly 60 million sequence reads produced between $\sim 10,800$ and $\sim 37,000$ total loci per

224 species (75 base pairs per locus, Table 1). The number of polymorphic loci scored in all samples

225 for each of the three species was 456, 1905, and 2508, for L. noctivagans, L. borealis, and $L$.

226 cinereus, respectively, and the total number of SNPs at these polymorphic loci was 835 for $L$.

227 noctivagans, 12,117 for L. borealis, and 8,236 for L. cinereus. All results reported below are

228 based on only these loci/SNPs sequenced in all individuals, as missing data, if analyzed, may

229 lead to underestimation of genetic diversity (Arnold et al. 2013). 
231 Genetic Isolation by Mortality Time

232 The AMOVA indicates that the vast majority $(>99 \%)$ of the molecular variance is

233 distributed within groups (Table 2). F $_{\text {ST }}$ values are generally low, indicating that there is little

234 genetic differentiation among groups of samples collected at different times of the year. Taken

235 together, the results of the AMOVA suggest that there is little differentiation as a function of

236 time in any of the three species. However, this analysis was based on an a priori grouping of

237 samples, so it could be the case that methods that do not require subjective a priori clustering of

238 samples would identify alternative partitions of genetic structure.

239 The probability of the data given various clustering levels (i.e., $K=1$ to $K=5$ ) was

240 estimated using STRUCTURE, with no prior information provided in terms of the time of

241 sampling. The overall $\log$ probability of the data was highest at $\mathrm{K}=1$ for both $L$. borealis and $L$.

242 cinereus (Fig. 2), suggesting this is likely the optimal clustering solution for these datasets. In

243 contrast, the highest likelihood for L. noctivagans was at $\mathrm{K}=2$. However, individual assignment

244 probabilities at $\mathrm{K}=2$ in $L$. noctivagans show a relatively constant amount of admixture across all

245 samples (Fig. S2) that is not consistent with a pattern expected from structured populations. As a

246 result, we see no strong evidence from the Structure data to reject a null hypothesis of a single

247 genetic population in any of these three datasets.

248 Results from the isolation by time analysis provided little support for a relationship

249 between genetic distance and time. While the null hypothesis of no relationship was rejected for

250 two of the three species (L. noctivagans and L. borealis), estimated slopes were small, and 95\%

251 confidence intervals for these slopes included zero in all cases (Table 3). In addition, for the two

252 species in which the null hypothesis was rejected based on the calculated p-value, the direction 
253 of the relationship associated with the point estimate of the slope varied, with a slightly negative

254 relationship between genetic distance and time for L. noctivigans, and a slightly positive

255 relationship for L. borealis.

256

257 Estimation of population and demographic parameters

258 Individual runs of fsc252 under each model consistently estimated a similar likelihood, 259 suggesting adequate searching of sample space and convergence on the maximum likelihood 260 parameters for each model. These analyses provided strong support for a growing population in 261 all three species, with essentially all of the relative AIC model weight assigned to a model of

262 increasing population size in each case (Table 4, Fig. 3). L. noctivagans had the lowest effective 263 population size estimates of the three species, but like the other two, was modeled as a growing 264 population, with a current (haploid) effective population size of 189,288 (CI 155,364-231,689)

265 that grew from an ancestral population of 7,001 (CI 11-37,656) starting 84,460 (CI 36,241-

$266285,149)$ generations in the past (Fig 3A, Table 5). For L. cinereus, parameter estimates

267 suggested that population growth began 156,608 (CI 133,774-479,831) generations ago, with an 268 ancestral effective population size of 17,023 (CI 11-40,216) growing to a current size of 830,623

269 (CI 730,470-884,046) individuals (Fig. 3B, Table 5). Like L. cinereus, estimates for $L$. borealis 270 population sizes were relatively large, with an ancestral population of 32,957 (CI 15-84,105)

271 individuals growing to 1,600,183 (CI 1,241,280-1,692,254) beginning 296,338 (CI 255,439-

272 888,838) generations ago (Fig 3C, Table 5). For all three species, estimates for the time at which 273 population growth began correspond approximately to the early to mid Pleistocene, depending 274 on generation length. Consistent with the trends in estimated current effective sizes from 
275 FastSimCoal252, the proportion of heterozygous loci was $0.088,0.182$, and 0.275 for $L$.

276 noctivagans, L. cinereus, and L. borealis, respectively.

277

278 Discussion

279

While DNA has been successfully isolated from bat wing tissue in samples collected

280 from wind facilities (e.g., Korstian et al. 2013), our study represents the first attempt to conduct

281 next-generation sequencing on these types of samples. Our results demonstrate the feasibility of

282 this approach, and suggest that genetic analyses of species impacted by wind facilities need not

283 be limited to microsatellite or mitochondrial barcoding sequence markers (Korstian, Hale \&

284 Williams 2015; Vonhof \& Russell 2015). We were able to genotype thousands of loci in each

285 species across all samples, and these data were used to assess the presence of population

286 structure, and to estimate demographic parameters in all three species.

287 We conducted several analyses that aimed to investigate the level of genetic structure in 288 our data. Our results provide little support for genetic structure in the data, including genetic 289 structure that may be partitioned as a function of the timing of mortality in our samples. For 290 example, AMOVAs indicate that very little of the genetic variance can be attributed to samples 291 collected at differing time points, and, similarly, clustering analyses in Structure provide little

292 evidence for population structure in the samples. We also conducted an isolation-by-time 293 analysis that is similar to analyses of genetic isolation by distance. In this analysis, the number

294 of days replaces quantitative measures of distance or environmental variables. When we

295 designed this analysis, we posited that (i) if populations were genetically structured in space, and

296 (ii) if bats from different populations were impacted by the wind facility at different times of the

297 year, then we might detect a signal of isolation by time. The overall lack of evidence for strong 
298 correlations between genetic distance and sampling time in each species is consistent with our

299 result of a lack of population structure from both the AMOVA and Structure analyses, and also

300 with recent results reported for L. borealis and L. cinereus (Korstian, Hale \& Williams 2015),

301 and separately for L. borealis (Vonhof \& Russell 2015).

302 Excoffier et al. (2013) introduced a composite likelihood method for estimating

303 population demographic parameters using the site frequency spectrum generated from genomic

304 datasets, and simulation testing indicates that their method (FastSimCoal2) provides accurate

305 estimates of parameters given data of the type collected here. Based on the results discussed

306 above, we modeled each dataset as a single random mating population in order to test for

307 evidence of population size change and to estimate demographic parameters for these bats.

308 Results for each species suggest that population size has been increasing since roughly the early

309 to mid Pleistocene, and that current population sizes are generally large.

310 As our manuscript was being prepared, two papers were published that also addressed the

311 questions of effective population size and demographic trends in two of the three species

312 investigated here. A linkage disequilibrium approach utilized by Korstian, Hale \& Williams

313 (2015) produced current Ne estimates for L. borealis and L. cinereus that were generally

314 uninformative, as most confidence intervals included infinity. As they point out, linkage

315 disequilibrium methods are not expected to be effective when true Ne values are high, as appears

316 to be the case for these species. In contrast, our current study, and previous work by Vonhof \&

317 Russell (2015), utilized coalescent approaches to generate estimates of current $\mathrm{Ne}$ in L. borealis.

318 Despite differences in the specific methods, geographic breadth of sampling, and types of genetic

319 markers, these two studies produced comparable estimates that were on the order of $10^{5}-10^{6}$ for

320 this species. Our study is the first to provide strong evidence for large effective sizes in $L$. 
321 cinereus and L. noctivagans. Estimates for this latter species were the lowest of all three groups,

322 but still exceeded $10^{5}$.

323 Estimates of demographic parameters such as Ne with model-based approaches like

324 FastSim Coal are dependent on the parameters included in the model used for the estimation

325 (Koopman \& Carstens 2010). By estimating parameters under different models, calculating AIC

326 scores for each, and ranking the models using model probabilities, we have removed some of the

327 uncertainty related to model choice from our analysis. However, additional uncertainty

328 associated with factors such as the assumed mutation rate necessitates that the absolute values of

329 any estimates are interpreted cautiously (see Methods for information on a sensitivity analysis

330 related to variation in the assumed mutation rate parameter).

331 For all three species, a model of population growth was a better fit to the data than

332 models of constant population size or population decline (see Table 4). Korstian, Hale \&

333 Williams (2015) and Vonhof \& Russell (2015) also provided evidence for population growth in

334 L. borealis, though estimates for the time at which growth began were later in these two studies

335 (late Pleistocene) than our dates, which tend to center on growth beginning in the early to mid

336 Pleistocene. Growth commencing at the late Pleistocene has been observed in a number of taxa,

337 and is likely associated with geographic range expansion from Pleistocene refugia. However, our

338 earlier dates may suggest that these bat populations are better characterized as having undergone

339 range shifts as opposed to range contractions during the Pleistocene. If true, this type of dynamic

340 may have been facilitated by the high vagility of these organisms.

341 It is important to note that the model comparisons and parameter estimates presented here

342 may not reflect very recent changes in population size associated with anthropogenic effects.

343 Our ability to detect such changes may be limited, in part due to the apparently large number of 
344 individuals and effective sizes characterizing these populations. Therefore, even though no

345 genetic data presented to date have demonstrated negative effects of anthropogenic impacts on

346 diversity in these bats, it is possible that this is a function of detectability as opposed to reflecting

347 a true lack of impact. As such, the data we have produced will provide a valuable baseline for

348 possible future genetic monitoring of these populations (Schwartz, Luikart \& Waples 2006).

349 Such monitoring could provide the opportunity to evaluate temporal trends in parameters that

350 may respond at different rates to population size changes, but that require sampling across

351 multiple time periods for inferring such trends (i.e. allelic diversity, see Allendorf et al. 2008).

352 While our study boasts a dataset that includes numbers of loci that far exceed any studies

353 performed on these bat species to date, the geographic scope of sampling in the current study

354 was restricted to a single Ohio wind farm. It is possible that our ability to detect population

355 structure in our samples is simply a result of sampling from a localized area, and that more

356 widespread geographic sampling would reveal distinct genetic populations. Work is ongoing to

357 obtain and analyze samples from a larger geographic region. However, given the differences in

358 the scope of sampling locations between our study and that of Vonhof \& Russell (2015; samples

359 ranged from Texas north to Ontario, but didn't include Ohio), the similarities in estimates of

360 effective population size for $L$. borealis between these two studies suggest that both may have

361 sampled from the same genetic population. This would suggest that samples from the Vonhof \&

362 Russell (2015) study are unlikely to represent separate components of a meta-population

363 structure that may have gone undetected in their analysis. In such a case, the samples from our

364 more localized region would likely have been characterized by a smaller Ne value than those in

365 their study that represented a larger geographic range. These results, in combination with the 
366 results addressing population structure above, generally seem to reflect a pattern of high gene

367 flow across large geographic areas in each of these highly vagile species.

368

369 Limits of genetics for sourcing migratory animals

370 In summary, we have demonstrated that RADseq can be a useful approach for generating

371 data from large numbers of genetic loci in tree bat populations. These data allowed for inferences

372 regarding long-term historical demographic processes, along with estimates of current effective

373 population sizes. However, while genetic data can at times be a powerful tool for identifying the

374 source of migratory animals (Ruegg et al. 2014), this requires that evolutionary processes have

375 acted to generate differentiation between the populations of interest. Similar to other recent

376 studies (Korstian, Hale \& Williams 2015; Vonhof \& Russell 2015), our data show no evidence

377 that this differentiation exists, which may be attributed to the high vagility of these species, along

378 with large effective population sizes. Therefore, while genetic data can provide useful

379 information on certain demographic aspects of these bats, and may be useful for long-term

380 temporal monitoring of genetic parameters of conservation relevance, alternative methods should

381 be investigated if management goals include sourcing samples. These alternatives may include

382 biomarkers such as stable isotopes (Voigt et al. 2012) or trace elements (Poesel et al. 2008), and

383 could serve as potentially powerful complements to genetic data for informing ecological and 384 conservation questions in tree bats.

388 We thank Jennifer Norris for support and for providing access to specimens, Ariadna Morales

389 Garcia for help with collecting tissue samples, and Jose Diaz for assistance with lab work. 
390

391

392

393

394

395

396

397

398

399

400

401

402

403

404

405

406

407

408

409

410

411

412

413

414

415

416

417

418

419

420

421

422

423

424

425

426

427

428

429

430

431

432

433

434

435

\section{References}

Allendorf FW, England PR, Luikart G, Ritchie PA, Ryman N. 2008. Genetic effects of harvest on wild animal populations. Trends in Ecology and Evolution 23:327-337.

Altizer S, Bartel R, Han BA. 2011. Animal migration and infectious disease risk. Science 331:296-302.

Arnett EB, Brown WK, Erickson WP, Fiedler J, Hamilton BL, Henry TH, Jain A, Johnson GD, Kerns J, Koford RR, Nicholson CP, O’Connell T, Piorkowski M, Tankersley R. 2007. Patterns of fatality of bats at wind energy facilities in North America. Journal of Wildlife Management 72:61-78.

Arnold B, Corbett-Detig RB, Hartl D, Bomblies K. 2013. RADseq underestimates diversity and introduces genealogical biases due to nonrandom haplotype sampling. Molecular Ecology 22:3179-3190.

Baerwald EF, D'Amours GH, Klug BJ, Barclay RMR. 2008. Barotrauma is a significant cause of bat fatalities at wind turbines. Current Biology 18:695-696.

Boulet M, Gibbs HL, Hobson KA. 2006. Integrated analysis of genetic, stable isotope, and banding data reveal migratory connectivity and flyways in the northern yellow warbler (Dendroica petechia; Aestiva group). Ornithological Monographs 61:29-78.

Cryan PM, Brown AC. 2007. Migration of bats past a remote island offers clues toward the problem of bat fatalities at wind turbines. Biological Conservation 139:1-11.

Cryan PM. 2008. Mating behavior as a possible cause of bat fatalities at wind turbines. Journal of Wildlife Management 72:845-849.

Davey JL, Blaxter MW. 2011. RADSeq: next-generation population genetics. Briefings in Functional Genomics 9:416-423.

Evanno G, Regnaut S, Goudet J. 2005. Detecting the number of clusters of individuals using the software STRUCTURE: a simulation study. Molecular Ecology 14:2611-2620.

Excoffier L, Smouse P, Quattro J. 1992. Analysis of molecular variance inferred from metric distances among DNA haplotypes: Application to human mitochondrial DNA restriction data. Genetics 131:479-491. 
436

437

438

439

440

441

442

443

444

445

446

447

448

449

450

451

452

453

454

455

456

457

458

459

460

461

462

463

464

465

466

467

468

469

470

471

472

473

474

475

476

477

478

479

480
Excoffier L, Lischer HEL. 2010. Arlequin suite ver 3.5: A new series of programs to perform population genetics analyses under Linux and Windows. Molecular Ecology Resources 10:564-567.

Excoffier L, Dupanloup I, Huerta-Sanchez E, Sousa VC, Foll M. 2013. Robust demographic inference from genomic and SNP data. PLOS Genetics 9:e1003905.

Faaborg J, Richard TH, Angela DA, Keith LB, Katie MD, Sidney AG Jr., Patricia H, Hobson KA, Jahn AE, Johnson DH, Latta SC, Levey DJ, Marra PP, Merkord CL, Nol E, Rothstein SI, Sherry TW, Sillett TS, Thompson III FR, Warnock N. 2010. Recent advances in understanding migration systems of New World land birds. Ecological Monographs 80, 348 .

Gautier M, Foucaud J, Gharbi K, Cezard T, Galan M, Loiseau A, Thomson M, Pudlo P, Derdelhue C, Estoup A. 2013. Estimation of population allele frequencies from nextgeneration sequencing data: pool-versus individual-based genotyping. Molecular Ecology 22:3165-3178.

Gutenkunst RN, Hernandez RD, Williamson SH, Bustamante CD. 2009. Inferring the joint demographic history of multiple populations from multidimensional SNP frequency data. PLoS Genetics 5:e1000695.

Horn J, Arnett EB, Kunz TH. 2008. Behavioral responses of bats to operating wind turbines. Journal of Wildlife Management 72:123-132.

Jameson JW, Willis CKR. 2014. Activity of tree bats at anthropogenic tall structures: implications for mortality of bats at wind turbines. Animal Behaviour 97:145-152.

Johnson GD, Erickson WP, Strickland MD, Shepherd MF, Shepherd DA, Sarappo SA. 2003. Mortality of bats at a large-scale wind power development at Buffalo Ridge, Minnesota. American Midland Naturalist 150:332-342.

Johnson GD. 2005. A review of bat mortality at wind-energy developments in the United States. Bat Research News 46:45-49.

Jonzén N, Hedenström A, Lundberg P. 2007. Climate change and the optimal arrival of migratory birds. Proceedings of the Royal Society of London B: Biological Sciences 274:269274.

Keightley PD. 2012. Rates and fitness consequences of new mutations in humans. Genetics 190:295-304.

Koopman MM, Carstens BC. 2010. Conservation genetic inferences in the carnivorous pitcher plant Sarracenia alata (Sarraceniaceae). Conservation Genetics 11:2027-2038. 
481

482

483

484

485

486

487

488

489

490

491

492

493

494

495

496

497

498

499

500

501

502

503

504

505

506

507

508

509

510

511

512

513

514

515

516

517

518

519

520

521

522

523

524

525

526
Korstian JM, Hale AM, Bennett VJ, Williams DA. 2013. Advances in sex determination in bats and its utility in wind-wildlife studies. Molecular Ecology Resources 13:776-780.

Korstian JM, Hale AM, Williams DA. 2015. Genetic diversity, historic population size, and population structure in two North American tree bats. Journal of Mammalogy DOI: http://dx.doi.org/10.1093/jmammal/gyv101

Kunz TH, Arnett EB, Erickson WP, Hoar AR, Johnson GD, Larkin RP, Strickland MD, Thresher RW, Tuttle MD. 2007. Ecological impacts of wind energy development on bats: questions, research needs, and hypotheses. Frontiers in Ecology and the Environment 5:315-324.

Kurta A. 1995. Mammals of the Great Lakes Region. The University of Michigan Press, Ann Arbor.

Loss SR, Will T, Marra PP. 2013. Estimates of bird collision mortality at wind facilities in the contiguous United States. Biological Conservation 168:201-209.

Nachman MW, Crowell SL. 2000. Estimate of the mutation rate per nucleotide in humans. Genetics 156:297-304.

Peterson BK, Weber JMN, Kay EH, Fisher HS, Hoekstra HE. 2012. Double digest RADseq: An inexpensive method for de novo SNP discovery and genotyping in model and non-model species. PLoS One 7:e37135.

Poesel A, Nelson D, Gibbs HL, Olesik J. 2008. Use of trace element analysis of feathers as a tool to track fine-scale dispersal in birds. Behavioral Ecology and Sociobiology 63:153-158.

Pritchard JK, Stephens M, Donnelly P. 2000. Inference of population structure using multilocus genotype data. Genetics 155:945-959.

Rappole JH, McDonald MV. 1994. Cause and effect in population declines of migratory birds. The Auk 111:652-660.

Ray DA, Feschotte C, Pagan HJT, Smith JD, Pritham EJ, Arensburger P, Atkinson PW, Craig NL. 2008. Multiple waves of recent DNA transposon activity in the bat, Myotis lucifugus. Genome Research 18:717-728.

Raymond M, Rousset F. 1995. GENEPOP (version 1.2): population genetics software for exact tests and ecumenicism. Journal of Heredity 86:248-249.

Rousset F. 2008. Genepop'007: a complete reimplementation of the Genepop software for Windows and Linux. Molecular Ecology Resources 8:103-106.

Ruegg KC, Anderson EC, Paxton KL, Apkenas V, Lao S, Siegel RB, DeSante DF, Moore F, Smith TB. 2014. Mapping migration in a songbird using high-resolution genetic markers. Molecular Ecology 23:5726-5739. 
527

528

529

530

531

532

533

534

535

536

537

538

539

540

541

542

543

544

545
Schwartz MK, Luikart G, Waples RS. 2006. Genetic monitoring as a promising tool for conservation and management. Trends in Ecology and Evolution 22:25-33.

Sovic MG, Fries AC, Gibbs HL. 2015. AftrRAD: a pipeline for accurate and efficient de novo assembly of RADseq data. Molecular Ecology Resources 15:1163-1171.

Voigt CC, Popa-Lisseanu AG, Niermann I, Kramer-Schadt S. 2012. The catchment area of wind farms for European bats: A plea for international regulations. Biological Conservation 153:80-86.

Vonhof MJ, Russell AL. 2015. Genetic approaches to the conservation of migratory bats: a study of the eastern red bat (Lasiurus borealis). PeerJ 3:e983 DOI 10.7717/peerj.983.

Weir BS. 1996. Genetic Data Analysis II: Methods for Discrete Population Genetic Data. Sinauer Assoc., Inc., Sunderland, MA, USA.

Weir BS, Cockerham CC. 1984. Estimating F-statistics for the analysis of population structure. Evolution 38:1358-1370. 


\section{Table $\mathbf{1}$ (on next page)}

Sequencing statistics.

Shown from left to right for each species are the number of samples, the average number of sequence reads per sample, the mean read depth for each locus, the total number of loci recovered, the number of total loci sequenced in all samples, and the number of polymorphic loci sequenced in all samples. 
1

\begin{tabular}{|l|l|l|l|l|l|l|}
\hline Species & Samples & $\begin{array}{l}\text { Mean } \\
\text { reads per } \\
\text { sample }\end{array}$ & $\begin{array}{l}\text { Mean } \\
\text { read } \\
\text { depth }\end{array}$ & $\begin{array}{l}\text { Loci } \\
\text { (total) }\end{array}$ & $\begin{array}{l}\text { Loci } \\
\text { (scored in } \\
\text { all } \\
\text { samples) }\end{array}$ & $\begin{array}{l}\text { Polymorphic } \\
\text { loci (scored in } \\
\text { all samples }\end{array}$ \\
\hline L. noctivagans & 37 & $2.49 \times 10^{5}$ & 11.71 & 10794 & 976 & 456 \\
\hline L. borealis & 53 & $3.86 \times 10^{7}$ & 36.58 & 37076 & 2287 & 1905 \\
\hline L. cinereus & 44 & $2.73 \times 10^{7}$ & 32.64 & 23920 & 3113 & 2508 \\
\hline
\end{tabular}

2 


\section{Table 2 (on next page)}

Analysis of Molecular Variance.

Shown for each species are the source of variation, the degrees of freedom, sum of squares, variance components, and percent variation. Also shown are the $F_{S T}$ values and probability that an $\mathrm{F}_{\mathrm{ST}}$ of this magnitude could occur by chance. Samples are grouped into temporal clusters (see Fig. 1) that formed the basis of the among and within group comparisons. 
1

\begin{tabular}{|c|c|c|c|c|}
\hline Species & \multicolumn{4}{|c|}{ L. noctivagans } \\
\hline $\begin{array}{l}\text { Source of } \\
\text { variation }\end{array}$ & d.f. & Sum of squares & $\begin{array}{l}\text { Variance } \\
\text { components }\end{array}$ & $\begin{array}{l}\text { Percent of } \\
\text { variation }\end{array}$ \\
\hline Among groups & 1 & 45.808 & 0.01371 & 0.03 \\
\hline Within groups & 72 & 3261.665 & 45.30091 & 99.97 \\
\hline Total & 73 & 3307.473 & 45.31461 & \\
\hline $\mathrm{F}_{\mathrm{ST}}$ & 0.0003 & \multicolumn{2}{|c|}{$\mathrm{P}$ (rand. value $>$ obs. value) } & 0.64907 \\
\hline Species & \multicolumn{4}{|c|}{ L. borealis } \\
\hline $\begin{array}{l}\text { Source of } \\
\text { variation }\end{array}$ & d.f. & Sum of squares & $\begin{array}{l}\text { Variance } \\
\text { components }\end{array}$ & $\begin{array}{l}\text { Percent of } \\
\text { variation }\end{array}$ \\
\hline Among groups & 2 & 828.907 & 1.48557 & 0.41 \\
\hline Within groups & 103 & 37295.706 & 362.09423 & 99.59 \\
\hline Total & 105 & 38124.613 & 363.57980 & \\
\hline $\mathrm{F}_{\mathrm{ST}}$ & 0.00409 & \multicolumn{2}{|c|}{$\mathrm{P}($ rand. value $>$ obs. value $)$} & 0.15347 \\
\hline Species & \multicolumn{4}{|c|}{ L. cinereus } \\
\hline $\begin{array}{l}\text { Source of } \\
\text { variation }\end{array}$ & d.f. & Sum of squares & $\begin{array}{l}\text { Variance } \\
\text { components }\end{array}$ & $\begin{array}{l}\text { Percent of } \\
\text { variation }\end{array}$ \\
\hline Among groups & 3 & 993.182 & 1.06590 & 0.34 \\
\hline Within groups & 86 & 26548.229 & 308.70034 & 99.66 \\
\hline Total & 89 & 27541.411 & 309.76624 & \\
\hline $\mathrm{F}_{\mathrm{ST}}$ & 0.00344 & \multicolumn{2}{|c|}{$\mathrm{P}$ (rand. value $>$ obs. value) } & 0.70674 \\
\hline
\end{tabular}

2 


\section{Table 3 (on next page)}

Isolation-by-time analysis.

Shown for each species are the slope and confidence interval of the best-fit linear relationship between genetic distance and collection time, along with the associated $p$-value. 


\begin{tabular}{|c|c|c|}
\hline Species & Slope $(95 \% \mathrm{CI})$ & $P$-value \\
\hline L. noctivagans & $-7.54 \times 10^{-6}\left(-5.73 \times 10^{-5}, 4.92 \times 10^{-5}\right)$ & 0.015 \\
\hline L. borealis & $5.68 \times 10^{-5}\left(-1.65 \times 10^{-5}, 1.33 \times 10^{-4}\right)$ & 0.002 \\
\hline L. cinereus & $-1.09 \times 10^{-4}\left(-3.05 \times 10^{-4}, 9.65 \times 10^{-5}\right)$ & 0.261 \\
\hline
\end{tabular}

2 


\section{Table 4(on next page)}

AIC model selection results for FastSimCoal analyses.

Two historical demographic models were compared for each of the three species: one simple model of constant population size, and a second model that allowed for a constant rate of growth or decline, the beginning of which is estimated. In all three species, the growth/decline model was strongly supported based on relative AIC model weights. 
1

\begin{tabular}{|l|l|l|l|l|}
\hline Species/Model & \# Parameters & Ln Likelihood & AIC & Akaike weight \\
\hline L. noctivagans & & & & \\
\hline Constant & 1 & -1556.879 & 7171.69 & $3.77 \mathrm{e}^{-31}$ \\
\hline Growth/Decline & 3 & -1525.59 & 7031.59 & 1 \\
\hline L. borealis & & & & \\
\hline Constant & 1 & -4906.422 & 22596.01 & $4.00 \mathrm{e}^{-235}$ \\
\hline Growth/Decline & 3 & -4671.156 & 21517.47 & 1 \\
\hline L. cinereus & & & & \\
\hline Constant & 1 & -7165.948 & 33002.41 & $3.41 \mathrm{e}^{-305}$ \\
\hline Growth/Decline & 3 & -6860.612 & 31600.29 & 1 \\
\hline
\end{tabular}

2

3 


\section{Table 5 (on next page)}

Parameter estimates from FastSimCoal analyses.

Parameter estimates from FastSimCoal analyses. Point estimates ( $\mathrm{Cl}$ ) of demographic parameters under the optimal growth/decline model for each of the species. Effective population sizes (number of chromosomes) for the current population and for the population prior to the beginning of population growth are given by 'N_CURR' and "N_ANCES", respectively. 'T_GRO_DEC' represents the time at which population growth or decline began (number of generations in the past; a model of population growth was supported in all three species). Confidence intervals were generated by parametric bootstrapping. 


\begin{tabular}{l}
1 \\
\begin{tabular}{|l|l|l|l|}
\hline Species & N_CURR & N_ANCES & T_GRO_DEC \\
\hline L. noctivagans & $189,288(155,364-231,689)$ & $7,001(11-37,656)$ & $84,460(36,241-285,149)$ \\
\hline L. borealis & $1,600,183(1,241,280-1,692,254)$ & $32,957(15-84,105)$ & $296,338(255,439-888,838)$ \\
\hline L. cinereus & $830,623(730,470-884,046)$ & $17,023(11-40,216)$ & $156,608(133,774-479,831)$ \\
\hline \\
4
\end{tabular} \\
\hline
\end{tabular}


Figure 1 (on next page)

Collection dates of bats chosen for genetic analyses.

Samples included in the study were selected in a manner that formed two temporal groups for L. noctivagans and L. cinereus (red squares and blue triangles, respectively) or three temporal groups for $L$. borealis (black circles). These clusters formed the basis for the AMOVA analysis. 
Collection Dates for Individual Bats During 2012

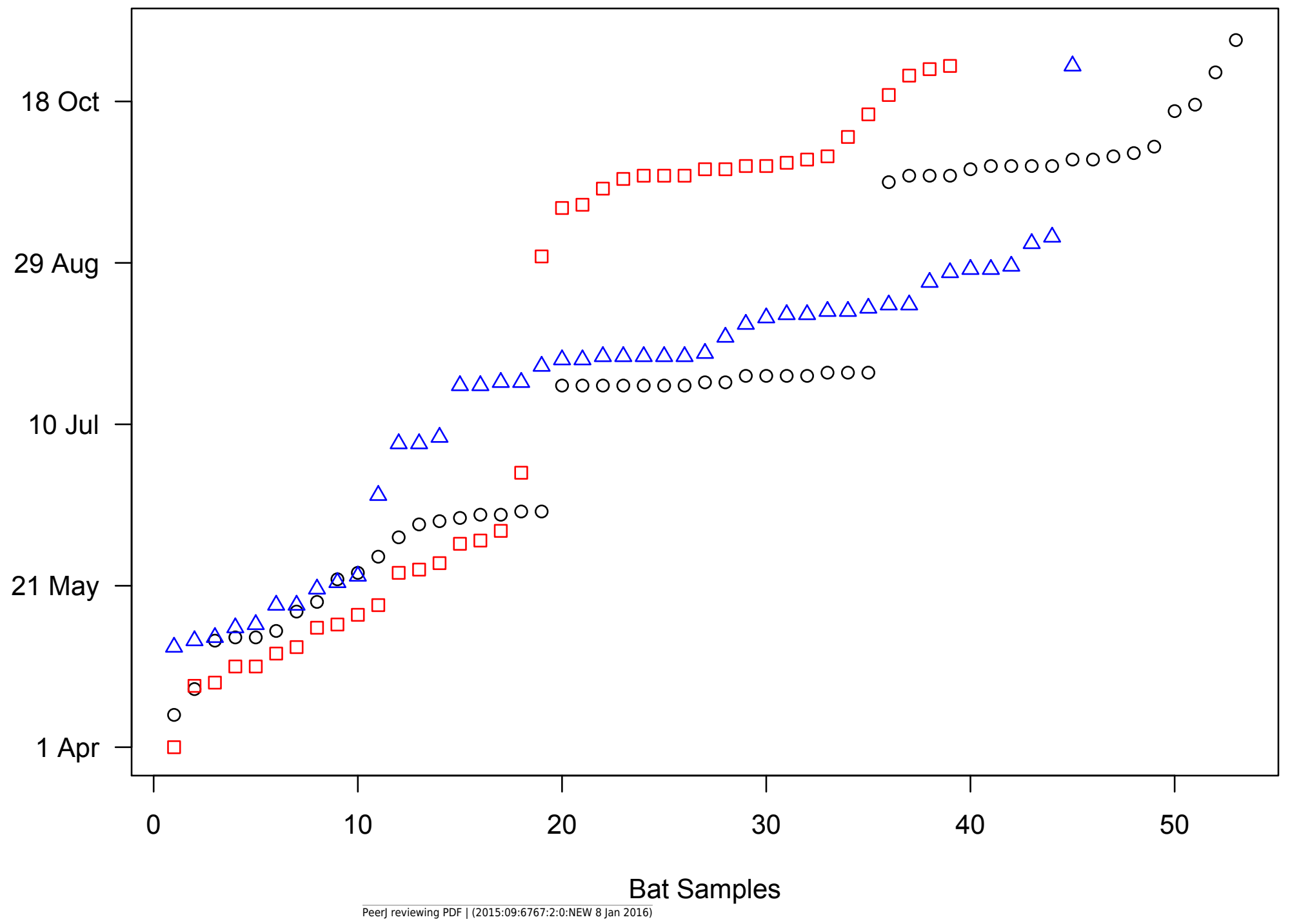


Figure 2 (on next page)

Genetic clustering results from STRUCTURE.

The mean $-\ln L$ for each species is shown for $k=1$ to $k=5$, where $k$ is the number of clusters assumed. The likelihoods for $L$. borealis and $L$. cinereus are highest at $K=1$, suggesting these datasets may best represent single panmictic groups. The likelihood for $L$. noctivagans was highest at $K=2$. However, individual assignment probabilities at $K=2$ failed to show a pattern consistent with genetic structure in this species, suggesting that it too may be best characterized as a single panmictic group. 
Silver-Haired

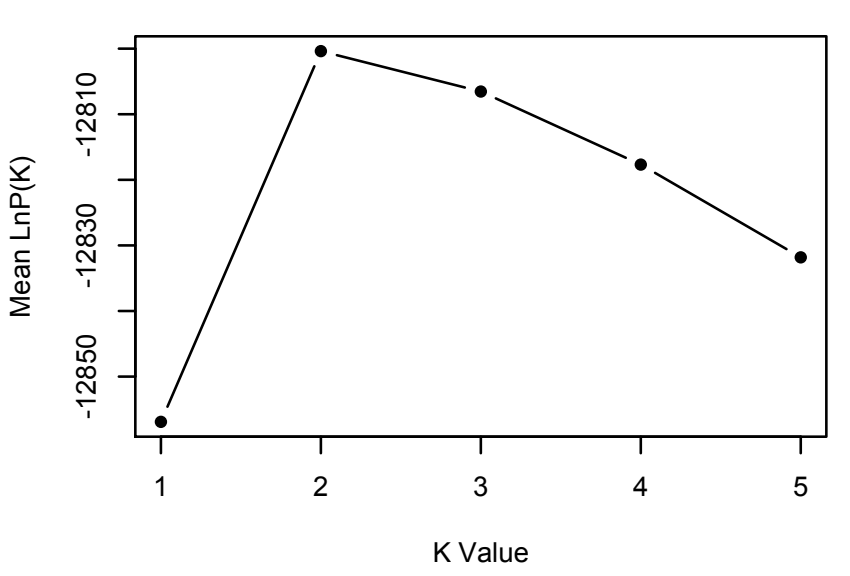

Hoary

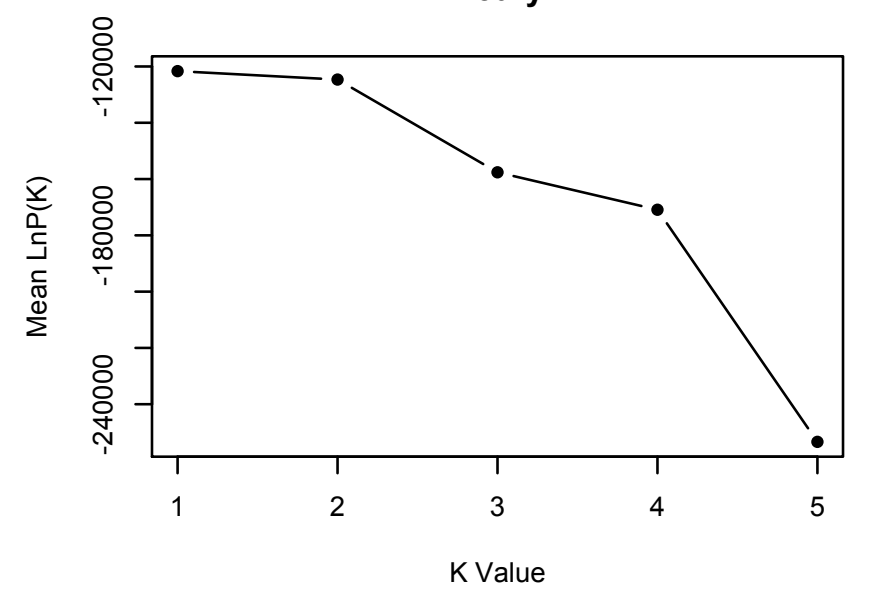

Eastern Red

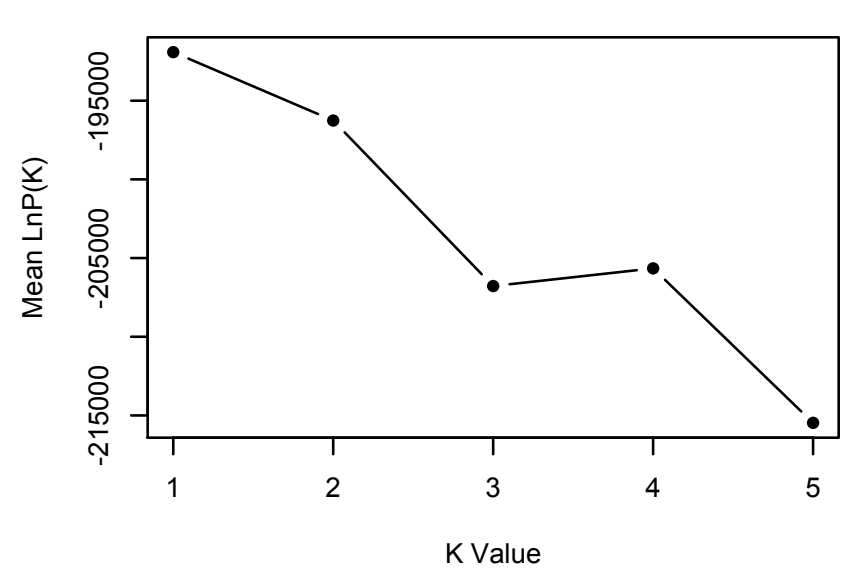




\section{Figure 3 (on next page)}

Demographic parameter estimates from FastSimCoal.

Results are based on a model of increasing population size, which was identified as the optimal model for each of the three species. Numbers inside the plots represent confidence intervals calculated for current effective population sizes (number of chromosomes). Numbers outside the plots represent confidence intervals for the time at which population growth began (number of generations in the past). Figures are scaled based on the point estimates of current effective size and time since growth began to represent the relative magnitudes of these parameters across the three species. 


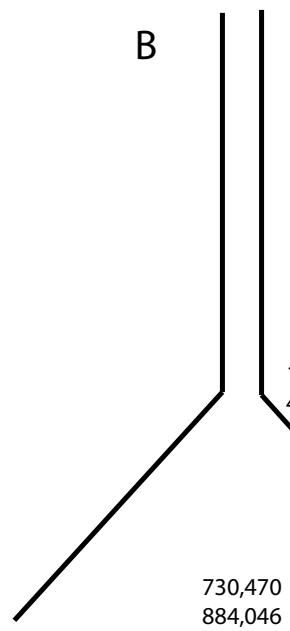

C borealis

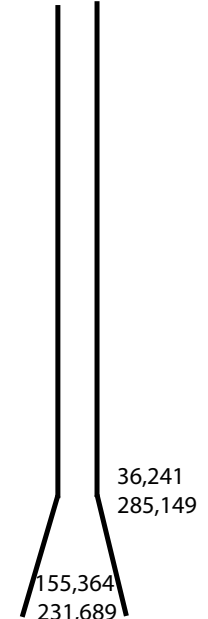

884,046

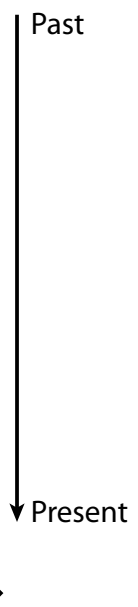

